\title{
MEDICAL_MAS: an Agent-Based System for Medical Diagnosis
}

\author{
Mihaela Oprea \\ University Petroleum-Gas of Ploiesti, Department of Informatics, \\ Bdul Bucuresti Nr. 39, Ploiesti, 100680, Romania
}

\begin{abstract}
The paper describes an agent-based system, MEDICAL_MAS, developed for medical diagnosis. The architecture proposed for the system includes mainly two types of agents: personal agents and information searching agents. Each type of personal agent corresponds to the humans involved in the medical diagnosis and treatment process (i.e. the patient, the physician, the nurse). The information searching agents are helping the personal agents to find information from the databases that can be accessed by the system (e.g. patients databases, diseases databases, medication databases etc). A case study is presented in the paper.
\end{abstract}

\section{Introduction}

A multi-agent approach is an attempt to solve problems that are inherently (physically or geographically) distributed, where independent processes can be clearly distinguished [1]. Such problems include, for example, decision support systems, networked or distributed control systems (see [2] for a recent review of such applications). The basis of this promising approach is supported by the main characteristics of intelligent agents and/or multi-agent systems, such as autonomy, flexibility, communication, coordination, security and privacy, mobility, adaptability, openness etc. Despite the fact that not all these characteristics are fully implemented at present, their potential application in the area of medicine is important. Due to the fast extension of the Internet and web-based systems in this area too, possible applications that we have identified include: the development of personal agents that assist physicians during their work, the management of a hospital (viewed as a virtual organisation), the patients monitoring and control, wireless applications for ambullance assistance, tutoring systems (e.g. in surgery, or in clinical guideline). At present, there are some agent-based systems or agent-based models for different medical specialities that have been reported in the literature (see e.g. [3], [4], [5], [6]).

Please use the following format when citing this chapter:

Oprea, M., 2009, in IFIP International Federation for Information Processing, Volume 296; Artificial Intelligence Applications and Innovations III; Eds. Iliadis, L., Vlahavas, I., Bramer, M.; (Boston: Springer), pp. 225-232. 
In this paper, it is presented an application of the multi-agent approach in the medical domain. MEDICAL_MAS is an agent-based system that is under development for medical diagnosis, and whose main purpose is to act as a decision support instrument for physicians during their activity of patients' diagnosis. The paper is organized as follows. Section 2 briefly presents the agent-based approach and some applications in the medical domain. The architecture of the agent-based system MEDICAL_MAS for diagnosis and treatment is described in section 3. A case study in the cardiology area is shown in section 4. The last section concludes the paper and highlights the future work.

\section{The Agent-based Approach and its Medical Applications}

The agent-based approach can significantly enhances our ability to model, design and build complex (distributed) software systems [7]. An agent can be viewed as a hardware or software entity that has properties such as autonomy and flexibility (social ability, reactivity, pro-activity). From the viewpoint of Artificial Intelligence (AI), an agent is a computer system that apart from the above mentioned properties that must be included in it, it is either conceptualized or implemented by using concepts that are more usually applied to humans (e.g. knowledge, belief, desire, intention, obligation, learning, locality, adaptation, believability, emotion) [8]. Other properties that an agent might have are: mobility, veracity, benevolence, rationality, etc. Also, an important remark is that an agent is embedded in an environment in which lives and interacts with other entities (e.g. agents, legacy software). So, an agent can be viewed as living in a society in which it has to respect the rules of that society. A multi-agent system (MAS) is a particular type of distributed intelligent system in which autonomous agents inhabit a world with no global control or globally consistent knowledge. The characteristics of multi-agent systems include: autonomy, communication, coordination, cooperation, security and privacy, mobility, openness, concurrency, distribution. Most of these characteristics need special approaches when designing and implementing a MAS. Usually, the MAS is based on a multi-agent infrastructure that enables and rules interactions, and acts as a middleware layer that support communication and coordination activities. A major characteristic of the agent technology is the high heterogeneity that means agent model heterogeneity, language heterogeneity, and application heterogeneity. This characteristic has to be manageable by using appropriate models and software toolkits.

Several projects are developing agent-based systems in the medical domain. One type of application is the agent-based modelling of a hospital, viewed as an electronic organization. An example of such framework for modelling a hospital as a virtual multi-agent organization is given in [6]. In some research projects intelligent agents are used for the distribution of human tissues or for the assignment of transplantable organs (see e.g. [6]). A MAS architecture for monitoring medical protocols was developed under the SMASH research project [9]. In [3] it is pre- 
sented the architecture of a MAS for monitoring medical protocols. Another application that could be modelled by the multi-agent approach is given by the automated clinical guideline in critical care (e.g. an extension of the SmartCare system described in [10], that uses a knowledge-based approach). Other potential applications that we have identified include the development of personal agents that assist physicians during their work, the management of a hospital (viewed as a virtual organisation), the patients monitoring and control, wireless applications for ambullance assistance, tutoring systems (e.g. in surgery). Practically, the main categories of applications are monitoring and control, diagnosis and treatment, planning and scheduling, tutoring. In this paper we shall focus on a multi-agent system dedicated to medical diagnosis and treatment, MEDICAL_MAS, that could act as a decision support instrument for physicians.

\section{The Architecture of the System MEDICAL_MAS}

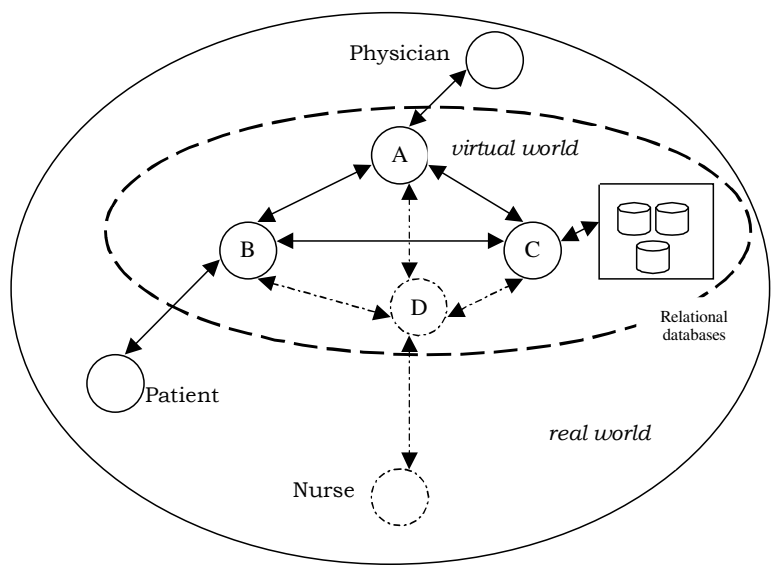

Fig. 1. The architecture of MEDICAL_MAS.

The architecture of the system MEDICAL_MAS is presented in Fig. 1. We have to note that the direct interactions that exist between the humans involved in a diagnosis process are not represented in Fig. 1, as we wanted to focus on the multiagent system architecture. The agents that compose the system are the virtual correspondences of the humans from the real world, involved in the diagnosis and treatment process, and some auxiliary agents. Thus, there is a physician agent (A), a patient agent (B), a nurse agent (D). All these three types of agents are personal agents that represent the interests of the humans whose representative they are in the virtual world. Other auxiliary agents are used for different purposes, such as for example, the information searching tasks. Agent $\mathrm{C}$ is an information searching 
agent whose task is to find information in the relational databases (patients, diseases, medications etc) that are available to MEDICAL_MAS. The architecture of the physician agent includes a rule-based reasoning mechanism and a knowledge base with decision rules for different diseases diagnosis and treatment. Each rule has associated a degree of confidence in that rule (called confidence factor).

We have designed the system for three possible scenarios of use: e-diagnosis (i.e. distant consultation), hospital use (for patients that are hospitalized), family physician.

In the case of distant consultation (what we've called $e$-diagnosis or $e$ consultation), the system could be used 24 hours from 24 hours. The patients could connect from their homes to the system and give to their personal agents (patient agents B) the description of the symptoms they have and ask for a quick diagnosis and treatment scheme (or at least medical advise) their personal or family physician or guard physician, actually the virtual representative of him, agent A, which is connected on-line (even when the physician is not on-line), and could take a quick decision before a real consultation will take place. We note that such a consultation could be scheduled also via the Internet. Potentially, in this scenario there is no need of agent D (the nurse agent). This is why in Fig. 1 the relations of agent $\mathrm{D}$ with the other agents are depicted by dashed lines. Thus, the MAS is composed only by agents A, B and C.

In the case of hospital use, all four types of agents are mandatory, A, B, C and D. Agent A will take the diagnosis decision based on the patient symptoms, on the historical data about the patient (given by agent $\mathrm{C}$ ), on the lab results (eventually, taken also electronically by agent $\mathrm{C}$ ) if asked, and on the expert knowledge he has. During this reasoning step it is used also a special type of expert knowledge (drugs' side-effects \& diseases medication interference), that referring to possible side-effects of drugs or of interference with other medications taken by the patient for other diseases he has. After the diagnosis and treatment decision made by the physician, the nurse agent $\mathrm{D}$ will receive from the physician agent the patient therapy and medication and will guide the nurse during their application.

In the case of family physician usage, the system is similar with that used in the first scenario, with the difference that the physician is always available on-line or at least, can quickly reply after an urgent e-consultation is asked. Basically, there are two possibilities of usage: the patient uses the system from home and has an on-line consultation with his family physician or the patient has a real consultation and during this his family physician uses the system MEDICAL_MAS to take a decision.

Figure 2 describes an interaction diagram. The PatientAgent asks for a consultation. Two situations may arise, either an agreement for the consultation when it is asked (this is the case represented in Fig. 2) or a negotiation for the scheduling of a consultation day and time agreed by both agents (Patient and Physician). During the consultation, the PhysicianAgent will ask the PatientAgent to tell the symptoms he has and also will ask the InfoSearchAgent to tell the historical data on the patient $\mathrm{P}$ (which are taken from the PatientsDataBases, PDB). Usually, more information are asked. For example, laboratory tests for the patient $\mathrm{P}$ (taken 
from the LaboratoryDataBases, LDB), more information about some diseases that $\mathrm{P}$ might have (taken from the DiseasesDataBases, DDB), and more information on medications and possible side effects and interferences with other medications of the diseases that patient $\mathrm{P}$ already has (this last information are taken from the MedicationsDataBases, MDB). All the databases used by the InfoSearchAgent are relational databases. When the PhysicianAgent receives all the asked information, he will use his reasoning mechanism and make a decision regarding the diagnostic (that with the highest confidence factor), the medication and the therapy that should be followed by the patient. In the example shown in Fig. 2, the PatientAgent is informed on the diagnostic, while the NurseAgent will be informed on the therapy and medication that patient $\mathrm{P}$ has to follow. This is the case when MEDICAL_MAS is used in a hospital.

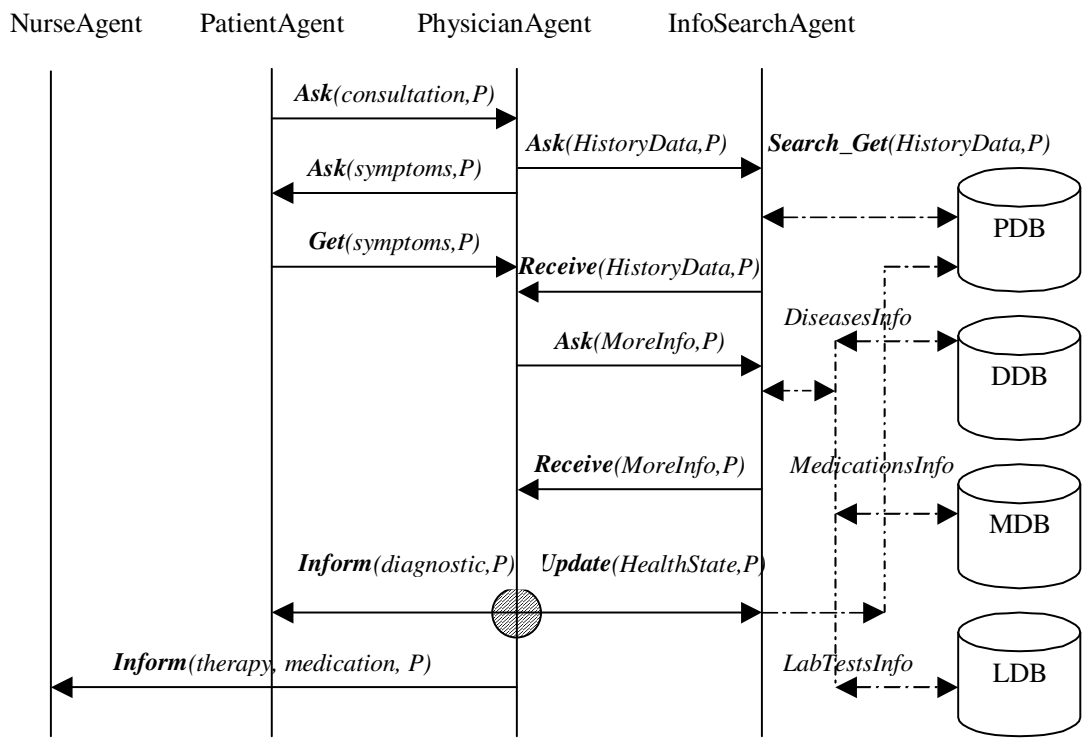

Fig. 2. Example of interaction diagram for the system MEDICAL_MAS.

\section{Case Study in Cardiology}

The system is developed in Zeus [11], and so far, we have implemented the ontology (Fig. 3 shows a screenshot with a part of the cardiology ontology), and we are currently developing a first version of the multi-agent system. 


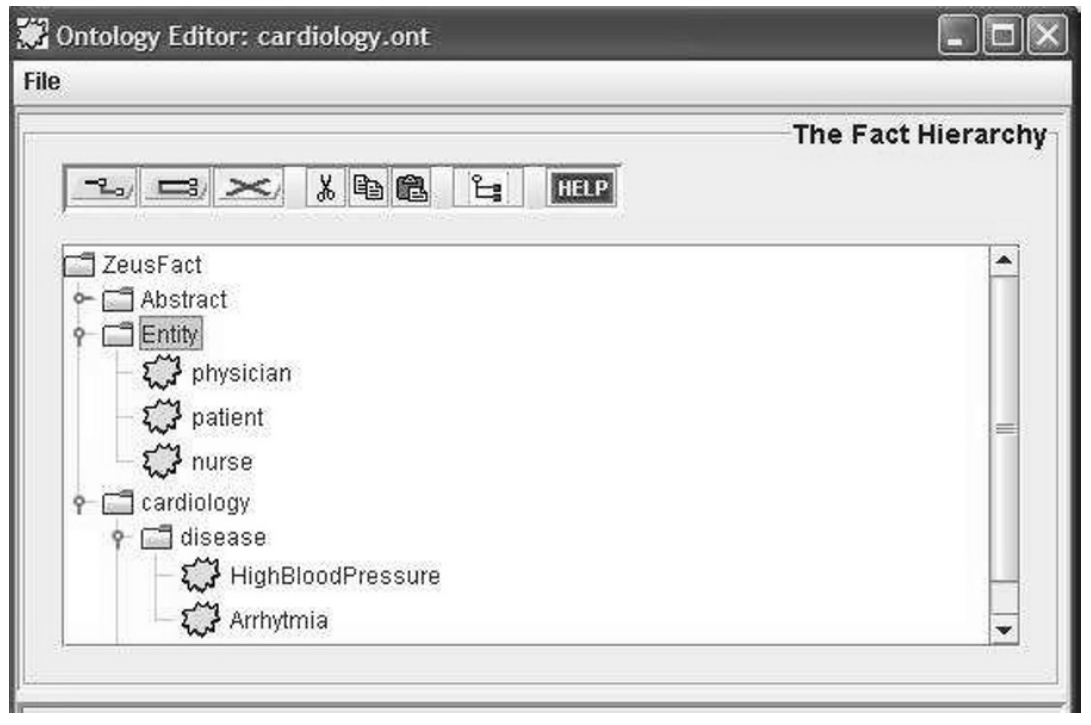

Fig. 3. Screenshot with the Zeus cardiology ontology (part of it).

In this section we shall analyse the use of MEDICAL_MAS in cardiology. Suppose the system is used in the scenario: personal physician specialized in cardiology. In Fig. 4 it is presented a simple example of the system run simulation.

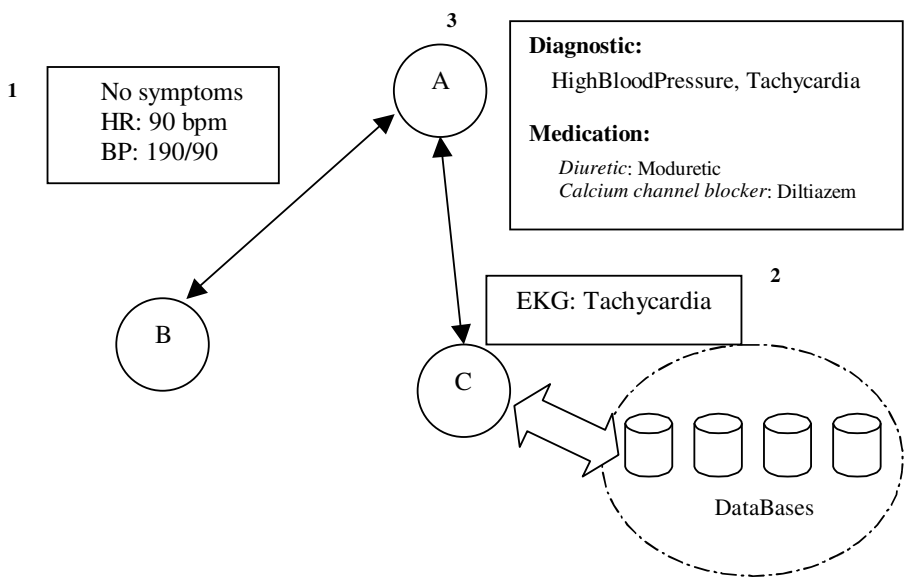

Fig. 4. Example of MEDICAL_MAS use in cardiology.

In the example we have taken the case of a patient that has no symptoms, but has problems with blood pressure. After the examination made by the physician with the use of the sphygmomanometer the collected data are the following:

$\{$ heart rate $(\mathrm{HR})=90 \mathrm{bpm}$, blood pressure $(\mathrm{BP})=190 / 90\}$ 
From the InfoSearchAgent (C) the PhysicianAgent (A) receives the result of an EKG Lab Test, which shows the presence of tachycardia. The result of the whole reasoning process of MEDICAL_MAS on the heart health state of patient (represented by agent B) gives the diagnostic of high blood pressure and tachycardia, and prescript a medication with two drugs: Moduretic (chosen so that it has no side effect on the potassium loss), and Diltiazem. As most of the antihypertensives have side-effects, all these interference rules are included in the knowledge base of the PhysicianAgent, A, so that the medication given to the patient will have minimum side-effects.

In the case of cardiology use, MEDICAL_MAS need to have included in the knowledge base of agent A the description of all possible diseases: arrhythmia, high blood pressure, cholesterol, heart attack, peripheral artery disease, congenital heart disease and so on (see e.g. [12]). Also, it should have a description of all categories of drugs. For example, in the case of high blood pressure there are several types of antihypertensives: diuretics, beta-blockers, ACE inhibitors, Angiotensin II receptor blockers, Calcium channel blockers etc. All the side-effects of the drugs need to be included in the knowledge base.

\section{Conclusion and Future Work}

The agent-based approach is one of the most efficient AI technologies that could be applied with success in the medical field, especially in the case of Internet and web-based applications extension in this area. The paper presented a multi-agent system for medical diagnosis, MEDICAL_MAS, that is under development, and was designed to act as a decision support instrument for physicians. So far, we have realized the analysis, and the design steps of the system development. Also, a first preliminary implementation in Zeus was made, and we have studied the use of the it in different scenarios. As a future work, we will end the implementation phase, and we shall experiment the system use in more different case studies.

The main advantage of using MEDICAL_MAS is given by the possibility of making the best decision regarding the diagnostic for a particular patient as well as giving the best medication in a specific context of the patient health state. We have to note that despite the fact that other artificial intelligence technologies (e.g. expert systems) have offered quite similar benefits in the medical domain, the multi-agent systems technology has the advantages (naming just few of them): of using distributed expertise knowledge (searched and collected on line by the agents), of being pro-active (e.g. taking the initiative to do some search on the web), and of being capable of communication. As time is an important resource for everyone, especially for physicians which are usually work overloaded, MEDICAL_MAS could offer them an efficient decision making instrument that can assist their work. 


\section{References}

1. Weiss, G. (ed.): Multiagent systems: a modern approach to distributed artificial intelligence, The MIT Press, Cambridge, Massachusetts (1999).

2. Oprea, M.: Applications of multi-agent systems, in: Reis, R. (ed.): Information Technology, Kluwer Academic Publishers, Boston Dordrecht London (2004) 239-270.

3. Alsinet, T., Béjan, R., Fernàndez, C., Manyà, F.: A Multi-Agent System Architecture for Monitoring Medical Protocols, Proceedings 4th International Conference on Autonomous Agents (2000) 499-505.

4. Gatti, N., Amigoni, F.: A Cooperative Negotiation Protocol for Physiological Model Combination, Proceedings 3rd International Joint Conference on Autonomous Agents \& Multi Agent Systems AAMAS (2004) 656-663.

5. Vàzquez-Salceda, J., Cortés, U., Padget, J.: Integrating the organ and tissue allocation processes through an agent-mediated electronic institution, Lecture Notes in Artificial Intelligence LNAI 2504, Springer Verlag, Berlin (2002) 309-321.

6. Vàzquez-Salceda, J., Dignum, F.: Modelling Electronic Organizations, in Mařík, V., Müller, J., Pěchouček, M. (eds.): Multi-Agent Systems and Applications III, Lecture Notes in Artificial Intelligence LNAI 2691, Springer Verlag, Berlin (2003) 584-593.

7. Wooldridge, M., Jennings, N.: Intelligent agents: theory and practice, The Knowledge Engineering Review, 10(2) (1995) 115-152.

8. Russel, S., Norvig, P.: Artificial Intelligence: A Modern Approach, Prentice Hall (1995).

9. SMASH project homepage: http://www.iiia.csic.es/Projects/smash/

10.Mersmann, S., Dojat, M.: SmartCare - Automated Clinical Guidelines in Critical Care, Proceedings of ECAI'04, IOS Press (2004) 745-749.

11. Zeus homepage: http://www.labs.bt.com/projects/agents/zeus/

12. http://americanheart.org 